BULL. AUSTRAL. MATH. SOC.

\title{
ALMOST COMPLEX STRUCTURES ON FOUR-DIMENSIONAL COMPLETE INTERSECTIONS
}

\author{
HOWARD HILLER*
}

\begin{abstract}
Suppose $X$ is a 4 -dimensional complete intersection in $C P^{p+4}$ of multidegree $d_{1}, \ldots, d_{p}$. We show that $X$ supports infinitely many almost complex structures for exactly $\&$ possible multidegrees. In particular, a hypersurface of degree $d$ in $C P^{5}$ admits infinitely many almost complex structures if and only if $d=2$ or 6 . This generalizes a result of $E$. Thomas [4] for $C P^{4}$. We give also some tables of possible Todd genera and a result for complex surfaces.
\end{abstract}

If $M$ is a $2 n$-dimensional differentiable manifold, then an almost complex structure (acs) on $M$ is a complex vector bundle $\omega$ satisfying ${ }^{\omega_{R}} \cong T M$, where $T M$ denotes the real tangent bundle of $M$. Hirzebruch [2] has posed the general problem of determining the possible total chern classes of acss on $M$.

E. Thomas [4] showed that $M=\mathbb{C P} P^{4}$ admits precisely 6 acss and wrote down their chern classes. If we view $C P^{4}$ as a degree 1 hypersurface $X_{4}(1)$ in $C P^{5}$, it is natural to consider Hirzebruch's question for $x_{4}(d), d>1$. If we define $N(d)=(d-2)(d-6)\left(5 d^{2}-8 d+8\right)$, the answer can be described in the following fashion:

Received 12 March 1984. "Partially supported by the Alexander von Humboldt stiftung.

Copyright Clearance Centre, Inc. Serial-fee code : 0004-9729/84 $\$ A 2.00+0.00$ 
THEOREM. (a) If $d \neq 2,6$, then $x_{4}$ (d) admits only finitely many acss each uniquely determined by their first Chern class. If $d$ is odd (resp. even) then the possible first Chern classes are the (resp. even) divisors of $N(d)$ (resp. $N(d) / 8)$.

(b) For all $d$, if $\omega$ is an acs over $X_{4}(d)$ and $c_{1}(\omega)=c_{1} x \in H^{2}\left(X_{4}(d)\right), c_{1}$ a non-zero integer, then the total Chern class of $\omega$ is given by:

$$
\begin{aligned}
c(\omega)=1+ & c_{1} x+\left(\frac{1}{2}\left(c_{1}^{2}+d^{2}\right)-3\right) x^{2}+ \\
& \left(\frac{1}{8}\left(c_{1}^{3}+\frac{N(d)}{c_{1}}+2 c_{1}\left(d^{2}-6\right)\right) x^{3}+\frac{x}{d} x^{4}\right.
\end{aligned}
$$

where $x$ denotes the Euler characteristic of $X_{4}(d)$ and $x$ is a generator of $H^{2}\left(x_{4}(d)\right)$.

(c) If $d=2,6 x_{4}(d)$ admit an acs with total Chem class (*), $c_{1}$ any non-zero integer. Furthermore for any integer $k$, $X_{4}(2)$ has an acs with Chern class $1-x^{2}+k x^{3}+3 x^{4}$ and $x_{4}(6)$ has an acs with Chem class $1-15 x^{2}+k x^{3}+435 x^{4}$.

There is a similar result for hypersurfaces in $\mathbb{C} P^{3}$ involving the midale-dimensional pairing that we describe below and a result for fourdimensional complete intersections.

Elsewhere we will consider Hirzebruch's problem for odd-dimensional complete intersections.

\section{Hypersurfaces in $\mathbf{C P} \mathbf{P}^{5}$.}

Up to diffeomorphism there is unique hypersurface $X_{n}(d)$ of degree $d$ in $C P^{n+1}$ defined by a single homogeneous polynomial of degree $d$. The topology of $X_{n}(d)$ is well-known and is described in Kulkarni-Wood [3]. We restrict our attention to $n=4$ but the facts we quote apply in complete generality. If $\alpha \in H^{2}\left(\mathbb{C P} P^{5}\right)$ denotes the first chern class of the hyperplane bundle $H$, then $x=i^{\star} \alpha$ generates $H^{2}\left(X_{4}(d)\right)$. The classes 
$x^{3}$ is $d$ times a generator of $H^{6}\left(X_{4}(d)\right)$ and $x^{4}$ evaluated on the fundamental class is $d$. The odd-dimensional cohomology groups are zero and $H^{2}\left(X_{4}(d)\right)$ is a free abelian group of rank $\beta_{2}=\frac{1}{d}\left\{(d-1)^{6}-1\right\}+2$, so that the Euler characteristic $x=B_{2}+4$. Finally we will need the characteristic classes of $X_{4}(d)$ that are easily computable from the basic relation

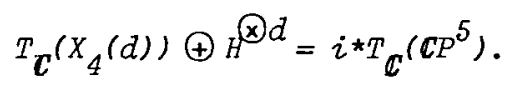

One gets:

$$
c\left(X_{4}(d)\right)=\frac{(1+x)^{6}}{1+d x}
$$

so that

$$
w\left(X_{4}(d)\right)=\left\{\begin{array}{lll}
(1+x)^{6} & \text { if } & d \equiv 0(2) \\
(1+x)^{5} & \text { if } & d \equiv 1(2) .
\end{array}\right.
$$

Similarly:

$$
p\left(x_{4}(d)\right)=1+\left(6-d^{2}\right) x^{2}+\left(d^{4}-6 d^{2}+15\right) x^{4}
$$

If $\omega$ is any acs over a $2 n$-manifold $M$, then (1) $c_{n}(\omega)=\chi(M)$ and (2) $c_{2 i}(\omega \oplus \bar{\omega})=p_{i}(M), 1 \leqslant i \leqslant n / 2$. Hence if $M$ is 8 -dimensional, we get

$$
\begin{cases}\text { (i) } & c_{4}(\omega)=x(M) \\ \text { (ii) } & p_{1}=c_{1}(\omega)^{2}-2 c_{2}(\omega) \\ \text { (iii) } & p_{2} \equiv c_{2}(\omega)^{2}-2 c_{1}(\omega) c_{3}(\omega)+2 c_{4}(\omega) .\end{cases}
$$

Substituting (i) and (ii) in (iii) and rearranging we obtain as in [4, Theorem 1.8] :

LEMMA 1.1. If $\omega$ is an acs over $\mu^{8}, c_{1}=c_{1}(\omega), c_{3}=c_{3}(\omega)$ then 
in $H^{8}(M ; \mathbb{Z})$

$$
8 \times(M)+p_{1}(M)^{2}-4 p_{2}(M)=8 c_{1} c_{3}-c_{1}^{4}+2 p_{1}(M) c_{1}^{2}
$$

It is now easy to compute the left-hand side of this identity for $M=X_{4}(d)$.

LEMMA 1.2. In $H^{8}\left(X_{4}(d)\right)$ :

$$
8 x+p_{1}^{2}-4 p_{2}=N(d) x^{4}
$$

where $N(d)=(d-2)(d-6)\left(5 d^{2}-8 d+8\right)$, as above.

Proof. From (1.0a) or the formula for the second Betti number above, one can compute $x=c_{4}\left(x_{4}(d)\right)=\left(d^{4}-6 d^{3}+15 d^{2}-20 d+15\right) x^{4}$. Combined with $(1.0 \mathrm{c})$ we get:

$$
8 x+p_{1}^{2}-4 p_{2}=5 d^{4}-48 d^{3}+132 d^{2}-160 d+96=N(d)
$$

after some easy computing.

COROLLARY 1.3. If $\omega$ is an acs over $x_{4}(d)$ and $c_{1}(\omega)=a x$, $c_{3}(\omega)=b x^{3}, a, b \in \mathbb{Z}$, then:

$$
a\left(8 b-a^{3}+2 a\left(6-d^{2}\right)\right)=N(d) .
$$

Proof. This is immediate from (1.1) and (1.2).

It remains to prove the converse of (1.3). According to Thomas [4, Theorem 3.1] it suffices to check

$$
S q^{2}\left(c_{1}(\omega)^{3}+c_{1}(\omega)^{2} w_{2}+c_{1}(\omega) w_{4}+c_{3}(\omega)\right)=0
$$

in $H^{8}\left(X_{4}(d) ; \mathbb{Z} / 2\right)$. For $d \equiv O(2)$ this is immediate as $x^{3} \equiv O(2)$.

If $d \equiv 1(2)$, we use $(1.0 \mathrm{~b})$ to observe that $w_{2} \equiv 1(2)$ and $w_{4} \equiv 0(2)$, so that the condition reduces to $b \equiv O(2)$. Hence it suffices to show:

LEMMA 1.4. If $a, d \equiv 1(2)$, then $a^{4}+N(d)+2 a^{2}\left(d^{2}-6\right) \equiv 0(16)$. 
Proof. From the proof of (1.2) we know

$$
N(d) \equiv 5 d^{4}+4 d^{2} \equiv 9(16)
$$

since $d^{2} \equiv 1(8)$ and $d^{4} \equiv 1(16)$. Similarly $a^{4} \equiv 1(16), 2 a^{2} d^{2} \equiv 2(16)$ and $-12 a^{2} \equiv 4(16)$. Hence $a^{4}+N_{d}+2 a^{2}\left(d^{2}-6\right) \equiv 1+9+2+4 \equiv 0(16)$.

Proof of Theorem. For $d$ odd, (a) follows from (1.3), (1.4) and Thomas' criterion (1.3.2). If $d$ is even, $\alpha$ is also even, so letting $a=2 k$ in (1.3.1) provides the result. (b) follows by computing $c_{2}, c_{3}$ explicitly from (1.0d). Finally (c) follows by observing that $d=2,6$ are roots of $N(d)$, so if $c_{1}=0, c_{3}$ is arbitrary, and if $c_{1} \neq 0$, it determines $c_{3}$ uniquely from (1.3.2).

REMARKS. 1 . The case $d=1$ of the theorem agrees with Thomas' result for $\mathbb{C P} P^{4}[4$, Theorem 3.2$]$.

2. It is easy to compute now the precise number of acss on $X_{4}(d)$. For example, if $d$ is odd then it is $2 d(N(d))$ where $d($. ) is the usual divisor function. (The 2 comes from allowing negative divisors.) See Table 1 for low values of $d$.

3. The formula

$$
\operatorname{Todd}(\omega)=\frac{1}{720}\left(-c_{4}+c_{1} c_{3}+3 c_{2}^{2}+4 c_{1}^{2} c_{2}-c_{1}^{4}\right)
$$

allows one to compute the possible Todd genera of acss on $x_{4}(d)$. See Table 2 for low values of $d$. If $d=2,6$ one can easily show:

COROLLARY 1.5. (a) A non-zero integer $t$ occurs as the Todd genus of an acs on the Klein quadric $x_{4}(2)$ if and only if $t=\frac{1}{12}\left(k^{4}-k^{2}\right)$, for some $k \in \mathbb{Z}$.

(b) A non-zero integer $t$ occurs as the Todd genus of an acs on $X_{4}(6)$ if and only if $t=\frac{1}{4}\left(k^{4}+15 k^{2}+8\right)$, for some $k \in \mathbb{Z}$.

2. Four-dimensional complete intersections

A complete intersection $x_{n}=x_{n}\left(d_{1}, \ldots, d_{n}\right) \subseteq \mathbb{C P} P^{n+r}$ 
is the transverse intersection of hypersurfaces of degrees $d_{1}, \ldots, d_{r}$ in $C P^{n+r}$. The ordered $r$-tuple $d_{1}<\ldots<d_{p}$ is called the multidegree of $x_{n} \subseteq \mathbb{C P} P^{n+r}$ and determines it up to diffeomorphism. The product $d_{1} \ldots d_{r}$ is the degree of $X$.

The results of $\$ 1$. admit generalization to 4 -dimensional complete intersections. We describe these here while omitting most of the details.

We will need some terminology from the theory of symmetric functions to define the analogue of $N(d)$. Fix a positive integer $r$ and view $d_{1}, \ldots, d_{p}$ as formal variables. If $I=\left(i_{1}, \ldots, i_{p}\right)$ is a partition of $k=i_{1}+\ldots+i_{r}$ then

$$
m_{I}\left(d_{1}, \ldots, d_{r}\right)=\left[d_{1}^{i_{1}} \ldots d_{p}^{i^{r}}\right.
$$

is the usual monomial symmetric function containing the indicated monomial. The complete elementary symmetric function $h_{k}$ is then given by:

$$
h_{k}=\sum_{I} m_{I}
$$

where $I$ varies over all partitions of $k$. The power-sum symmetric function is given by:

$$
P_{k}=\sum_{i=1}^{r} d_{i}^{k}
$$

We now introduce the following symmetric polynomial

$$
\begin{aligned}
N_{r}\left(d_{1}, \ldots, d_{r}\right)= & \left(8 h_{4}-2 p_{4}-p_{2}^{2}\right)-8(r+5) h_{3}+ \\
& 4(r+5)(r+4) h_{2}+2(r+5) p_{2}- \\
& 8\left(\frac{r+5}{3}\right) h_{1}+8\left(\frac{r+5}{4}\right)-(r+5)^{2}+2(r+5)
\end{aligned}
$$

We leave it to the reader to check the analogue of (1.2), that $8 \mathrm{x}+p_{1}^{2}-4 p_{2}=N_{r}\left(d_{1}, \ldots, d_{r}\right)$ in $H^{8}\left(X_{4}\left(d_{1}, \ldots, d_{r}\right)\right)$, in particular, $N_{1}=N$. It is now easy to describe a parametrization of the acss over 
$X_{4}\left(d_{1}, \ldots, d_{p}\right)$ as in section 1

It remains only to determine which multidegrees support infinitely many acss or equivalently acss with zero first chern class. These correspond precisely to the integral zeros of $N_{r}\left(d_{1}, \ldots, d_{r}\right)$. It is possible to check the following.

PROPOSITION 2.1. The following multidegrees support infinitely many acss:

$\begin{array}{ccccc}\underline{r=1} & \frac{r=2}{2} & \frac{r=3}{r=4} & \underline{r=5} \\ 6 & 2,5 & 2,2,4 & 2,2,2,3 & 2,2,2,2,2 \\ & 3,4 & 2,3,3 & \end{array}$

and these are all of them.

\section{Hypersurfaces in $\mathbf{C} \mathbf{P}^{3}$}

The main result is:

THEOREM 3.1. The almost complex structures on $X_{2}(d) \subseteq \mathbb{C P} P^{3}$ correspond to the elements $\beta \in H^{2}\left(X_{2}(d) ; \mathbb{Z}\right)$ satisfying $B \equiv d x(2)$ and $\beta^{2}=(d-4)^{2} x^{2}$.

Proof. According to Wu [5] and Ehresmann [1] acss on a 4-manifold $M$ are classified by elements $\beta \in H^{2}(M)$ satisfying $\beta \equiv w_{2}(M)(2)$ and $\beta^{2}-2 x(M)=p_{1}(M)$. If $M=X_{2}(d)$, we have $p_{1}=\left(4-d^{2}\right) x^{2}$, $X(M)=c_{2}(M)=\left(d^{2}-4 d+6\right) x^{2}$ and $w_{2} \equiv d(2)$; so the result follows.

COROLLARY 3.2. If $X$ is a smooth $K 3$ surface, then acss on $X$ correspond to even null vectors in $\mathbb{Z}^{22}$ with respect to the form $2 E_{8} \oplus 3\left(\begin{array}{ll}0 & 1 \\ 1 & 0\end{array}\right)$

Proof. $X$ is realized by the smooth quartic hypersurface in $\mathbb{C} P^{3}$. 


\begin{tabular}{cc} 
degree $d$ & number of acss on $X_{4}(d)$ \\
\hline 1 & 6 \\
2 & $\infty$ \\
3 & 8 \\
4 & 8 \\
5 & 12 \\
6 & $\infty$ \\
7 & 8 \\
8 & 24 \\
9 & 32 \\
10 & 16 \\
11 & 48 \\
12 & 32
\end{tabular}

TABLE 1 
Chern classes of almost complex structures on $X_{4}(d)$.

\begin{tabular}{|c|c|c|c|c|}
\hline $\pm c_{1}$ & $c_{2}$ & $\pm c_{3}$ & $c_{4}$ & Todd genus \\
\hline \multicolumn{5}{|c|}{$d=3$} \\
\hline 1 & 2 & -10 & 9 & 0 \\
\hline 3 & 6 & 2 & 9 & 1 \\
\hline 29 & 422 & 3070 & 9 & 5565 \\
\hline 87 & 3786 & 82378 & 9 & 447931 \\
\hline \multicolumn{5}{|c|}{$d=4$} \\
\hline 2 & 7 & -8 & 47 & 1 \\
\hline 4 & 13 & 11 & 47 & 6 \\
\hline 14 & 103 & 376 & 47 & 441 \\
\hline 28 & 397 & 2813 & 47 & 6566 \\
\hline \multicolumn{5}{|c|}{$a=5$} \\
\hline 1 & 10 & -30 & 165 & 1 \\
\hline 3 & 14 & 6 & 165 & 6 \\
\hline 9 & 50 & 130 & 165 & 126 \\
\hline 31 & 490 & 3870 & 165 & 12501 \\
\hline 93 & 4334 & 100986 & 165 & 978306 \\
\hline 279 & 38930 & 2716030 & 165 & 78934630 \\
\hline
\end{tabular}

TABLE 2 


\section{References}

[1] C. Ehresmann, "Sur les variétés presque complexes", Proc. Int. Cong. Math. 2 (1950), 412-419.

[2] F. Hirzebruch, "Some problems on differentiable and complex manifolds", Ann. of Math. (2) 60 (1954), 213-236.

[3] R. Kulkarni and J. Wood, "Topology of non-singular complex hypersurfaces", Advances in Math. 35 (1980), 239-263.

[4] E. Thomas, "Complex structures on real vector bundles", Amer. J. Math. 89 (1967), 887-908.

[5] W. T. Wu, "Sur les espaces fibrés", Actual. scient. ind. (1952), 1183.

Mathematiches Institut

Universitat Gottingen

D-4500 Gottingen

West Germany

and

Department of Mathematics

Columbia University

New York, N.Y. 10027

U.S.A. 